\title{
Effect of desk materials on affective states and cognitive performance
}

\author{
Dean Lipovac ${ }^{1,2^{*}}$ (D) Nastja Podrekar ${ }^{1,3}$ (D) Michael D. Burnard ${ }^{1,2}$ (D) and Nejc Šarabon ${ }^{1,3}$ (D)
}

\begin{abstract}
Visual and tactile properties of materials can influence human emotional and cognitive functioning. Existing studies indicate that exposure to wood may lead to more favourable outcomes than contact with other common materials, but evidence is limited. We measured affective states and cognitive performance in 16 participants before and after each spent $15 \mathrm{~min}$ at 10 desks with differing top surfaces. Desk surfaces were made of untreated, oiled, or lacquered spruce or oak solid wood, laminated or oak-veneered particleboard, glass, and mineral-filled thermoplastic composite. The results indicate that cognitive performance and affective states of participants did not differ between the desk surfaces. It appears that exposure to a relatively small wooden surface does not significantly influence affective and cognitive outcomes. Incorporating larger amounts of wood coverage and a more demanding cognitive task would probably increase the chances of capturing the potential effects of wood exposure on human affective states and cognitive performance.
\end{abstract}

Keywords: Wood, Materials, Emotion, Cognition, Attention

\section{Introduction}

An increasing body of evidence demonstrates that when people are exposed to natural environments or elements of nature, such as plants or water, certain indicators of human stress and well-being tend to improve, including affective states, cognitive performance, and physiological arousal [1]. These observations are typically explained through the stress reduction theory [2], emphasizing physiological and affective improvements in response to nature exposure, or/and attention restoration theory, highlighting restoration of cognitive capabilities $[3,4]$. The findings in this field urge building designers to provide connection to nature in the built environment, where people spend most of their time [5]. Indeed, indoor nature exposure can be seen as a health-promoting framework [1].

Pleasant flower aromas, lush greenery, and water walls are some of the ways in which nature can be brought

\footnotetext{
*Correspondence: dean.lipovac@innorenew.eu

${ }^{1}$ InnoRenew CoE, Livade 6, 6310 Izola, Slovenia

Full list of author information is available at the end of the article
}

indoors. The modern built environment, however, does not only need to be a nature surrogate, but has to offer practical solutions, which cannot be directly constructed from most elements of nature. As a result, most elements of nature have limited capabilities of bringing indoor spaces closer to the natural environment.

In contrast, wood is a material that can be used in most indoor furnishings [6]. It is perceived as more natural than other common building materials and, accordingly, indoor spaces containing more wood are rated as more natural than their counterparts [6-8]. The current research inspects how humans can be affected through visual, tactile, and olfactory wood stimulation [9-11]. Of these, visual and tactile stimulation of wood might be easier to implement on a wider scale, as it seems challenging for indoor wood furnishings to provide longterm olfactory stimulation that is as intense as the one delivered in the experiments observing positive findings [12].

Altogether, not many studies have investigated the effects of tactile and visual exposure to wood. The existing findings demonstrate that touching wood can
SpringerOpen

(c) The Author(s) 2020. This article is licensed under a Creative Commons Attribution 4.0 International License, which permits use, sharing, adaptation, distribution and reproduction in any medium or format, as long as you give appropriate credit to the original author(s) and the source, provide a link to the Creative Commons licence, and indicate if changes were made. The images or other third party material in this article are included in the article's Creative Commons licence, unless indicated otherwise in a credit line to the material. If material is not included in the article's Creative Commons licence and your intended use is not permitted by statutory regulation or exceeds the permitted use, you will need to obtain permission directly from the copyright holder. To view a copy of this licence, visit http://creativeco mmons.org/licenses/by/4.0/. 
improve certain indicators of stress and well-being when compared to the effects of the tactile experience of other everyday materials, such as aluminium or marble $[9,13$, 14]. These studies showed that certain indicators of physiological arousal decrease after a short tactile exposure to wood (typically $90 \mathrm{~s}$ ). Other studies observed similar physiological outcomes when investigating visual wood exposure [15-17], while certain studies that combined visual and olfactory wood stimulation detected improved affective states in the wooden environment [18-20].

However, overall evidence for positive effects of tactile or visual wood exposure is limited, as it is not clear if the effects of wood exposure can extend beyond the initial brief period being observed in several existing studies. Additionally, it is not known if wood exposure, in the absence of olfactory stimulation, can influence not only (often ambiguous) physiological outcomes [21], but also other indicators of stress and well-being, such as affective states and cognitive performance.

Six studies were found that have investigated the effects of wood exposure on affective states [8, 18-20, 22, 23]. Five of these used the Profile of Mood States (POMS) measurement scale, which was devised to assess psychiatric outcomes and may be less sensitive to changes in affective states in different contexts [24], including in investigations of human responses to natural environments. In addition, due to its length (65 items in its original form), the questionnaire is less appropriate for repeated administrations typical in restoration studies, as it can encourage unvarying responses that can lead to misleading similarities in results between measurements [25]. A carefully chosen measure (fitting to the study context) can increase the chances of capturing potential changes in affective states [24].

We found only two studies that examined cognitive performance following the exposure to wood $[16,17]$. One of these did not report the results due to issues with the nature of the employed task [17]. In the other study, which did not detect differences between the tested environments, cognitive performance was not assessed prior to exposure to the experimental settings [16]. This excludes the possibility of detecting baseline differences in performance and it does not induce cognitive fatigue that might be important in finding differences in restoration between environments [26, 27]. In addition, the type of the deployed task and the length and timing of its administration can be decisive in detecting restoration of cognitive performance [26-28]. These observations indicate that greater attention is needed in selecting and administering cognitive tasks in restoration research.

In addition to knowledge gaps related to affective states and cognitive performance, it is not clear if and how different wooden materials influence indicators of well-being. The existing studies incorporated various wood types, colours, and species, from birch woodveneered furniture [16] to oak and walnut-veneered furniture [17] and spruce and fir solid wood [18]. These studies typically compared the effects of a wooden versus a control material, but not different wooden materials with each other. As visual and tactile properties of wood differ between wood species and treatments as well as between solid and processed wood, different wooden materials might lead to diverse human responses.

The scarcity of research and the specific methodological approaches identified in many existing studies lead to several gaps in knowledge that we investigated in the present study. The objective of the study was to investigate the effects of tactile and visual exposure to (untreated and treated) wood, glass, and mineral-filled thermoplastic composite (MFTC) desk materials on cognitive performance and affective states, while incorporating a fitting measure of affective states and including an assessment of cognitive performance prior to the subjects' exposure to the experimental settings.

We hypothesized that (1) wooden materials compared to the non-wooden materials and (2) untreated wooden materials compared to treated wooden materials will:

\section{a. Influence affective states}

1. Increase the pleasure dimension.

2. Decrease the arousal dimension.

b. Improve cognitive performance

3. Increase the proportion of correct answers on the cognitive task.

4. Decrease the mean response time in correct answers.

5. Decrease the differences in mean response time between congruent and incongruent task trials (Simon effect).

\section{Materials and methods Participants}

A convenience sample of 16 volunteer subjects $\quad($ mean $=25.88$ years, standard deviation $(S D)=3.98$ years; 10 women) participated in the study. Subjects belonged to the social network of the first two authors. They were eligible to participate in the study if they did not present with health issues or other characteristics that would make completing the study tasks difficult (e.g. very poor vision). A large majority of the invited people agreed to participate in the study; the refusals were generally related to the lack of time. In general, 
participants were not expected to possess in-depth knowledge on materials that could substantially influence the results, even though that the educational background of two participants included wood science. Subjects did not receive any compensations for participating in the study. Before the experiment, participants signed an approved informed consent waiver that provided them information about study purpose and procedure, participant rights, and data management. The study protocol and testing procedures (ClinicalTrials.gov identifier: NCT03733366) were approved by the National Medical Ethics Committee of Slovenia (No. 0120-631/2017/2) and the research was carried out in compliance with the Oviedo convention.

\section{Sample size and power}

Statistical power for Friedman tests was computed by calculating the power of repeated measures analysis of variance and transforming the resulting value [29]; the study was underpowered ( $1-\beta$ (type II error probability $)=0.52$, effect size: Cohen's $f=0.8)$. Statistical power for Wilcoxon tests was calculated empirically using Monte Carlo simulation [30]. The empirical power of

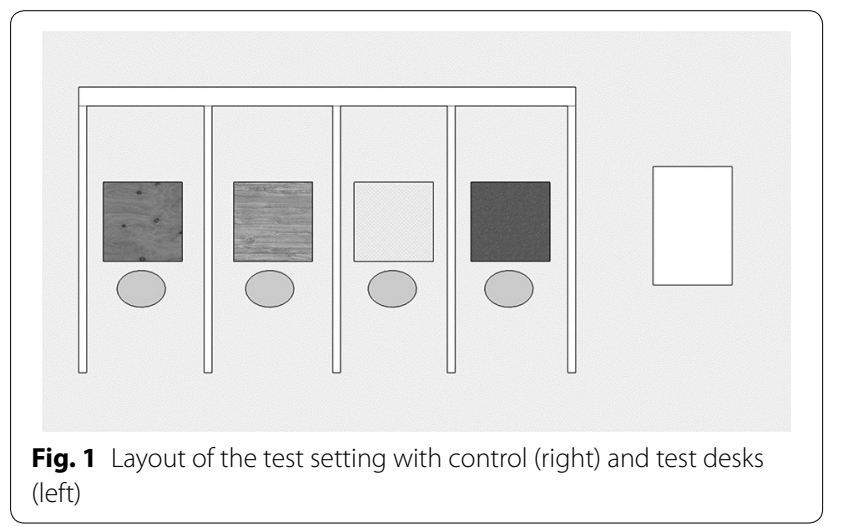

these tests is 0.88 with a large effect size (Cohen's $f=0.8$ ). The effect size in this power calculation indicates the probability that $X<Y$, where $X$ and $Y$ are random observations from the compared data. When testing a location shift hypothesis, the null hypothesis is an effect size of 0.5 and effect size increases as the probability approaches 0 and $1[30]$.

\section{Test setting}

The experiment was performed at the kinesiology and ergonomics laboratory of the University of Primorska in Izola, Slovenia. The layout of the test setting is presented in Fig. 1. Four desk frames were prepared from solid spruce wood and were positioned close to a white wall (participants were seated to face the wall). White drapes were put in between the desk frames, so the subjects participating in the experiment simultaneously could not see each other. Next to them was a control desk $(80 \times 120 \mathrm{~cm})$ that was covered with a plain white tablecloth and had no chairs (participants were standing). The control desk was not divided in sections and when many simultaneous sessions were running, participants were able to see each other. The indoor temperature and relative humidity were measured several times per day; the mean values were $23.58{ }^{\circ} \mathrm{C}(\mathrm{SD}=0.69)$ and $37.40 \%$ $(\mathrm{SD}=7.05)$, respectively. The experiment took place in November and December 2018. Most of the testing was conducted in first half of the day during weekdays, but sessions were sometimes executed later in the day and on weekends.

\section{Desk materials}

Desk surfaces were prepared from 10 different materials (with dimensions $80 \times 80 \mathrm{~cm}$; thickness varied by material), listed in Table 1 and presented in Additional file 1: Figures S1 to S9. Eight surfaces were manufactured from wood; six from solid wood (and were either untreated,

Table 1 Desk materials used in the study

\begin{tabular}{lll}
\hline Material & Surface treatment & Material thickness \\
\hline Spruce & Untreated & $2 \mathrm{~cm}$ \\
Spruce & Oil & $2 \mathrm{~cm}$ \\
Spruce & Lacquer & $2 \mathrm{~cm}$ \\
Oak & Untreated & $2 \mathrm{~cm}$ \\
Oak & Oil & $2 \mathrm{~cm}$ \\
Oak & Lacquer & $2 \mathrm{~cm}$ \\
Oak (veneer) & Untreated & $2 \mathrm{~cm}$ \\
Imitation wood laminate & - & $2 \mathrm{~cm}$ \\
Glass (on laminate) & - & $1.5 \mathrm{~cm}$ (glass) $+0.5 \mathrm{~cm}$ (laminate) \\
MFTC & - & $0.8 \mathrm{~cm}$ (MFTC) $+1.2 \mathrm{~cm}$ (particleboard)
\end{tabular}


oiled, or lacquered) and two were wood composites. The two non-wood materials were glass and a commercially available MFTC $\left(\right.$ Kerrock $\left.^{\circledR}\right)$. An example is presented in Fig. 2.

\section{Measures of affective states, cognitive performance, and material assessment}

Subjects completed an affective state assessment and a cognitive performance task that were presented with Tatool, an open-source test platform [31]. After an introduction to the experiment, the test procedure was automated, including breaks and instructions.

\section{Pleasure and arousal single-item scales}

Affective states were examined with two single-item scales assessing the states of pleasure and arousal [32]. As it is not clear which specific affective states can be induced by wood exposure, including any specific measure increases the chances of not detecting other affective changes [33]. For this reason, we used the scales capturing the broad state of core affect-simplest consciously accessible feelings [33]. In addition, the testing with the single-item scales is brief and thus especially useful when many assessments are carried out in a short period of time [32], as is the case in our protocol. Despite the brevity, these measures have proven to be reliable and valid [32, 34]. The two administered items asked: "How pleasant/activated do you feel at this moment?". Participants provided answers on a 9-point Likert-type scale $(1=$ especially unpleasant/inactivated, $5=$ neutral, $9=$ especially pleasant/activated).

\section{Simon task}

Cognitive performance was assessed with the Simon task that captures inhibitory control-the ability to override the urge to an internal predisposition or an external lure $[35,36]$. We based our task selection on the findings of a recent review demonstrating that exposure to nature

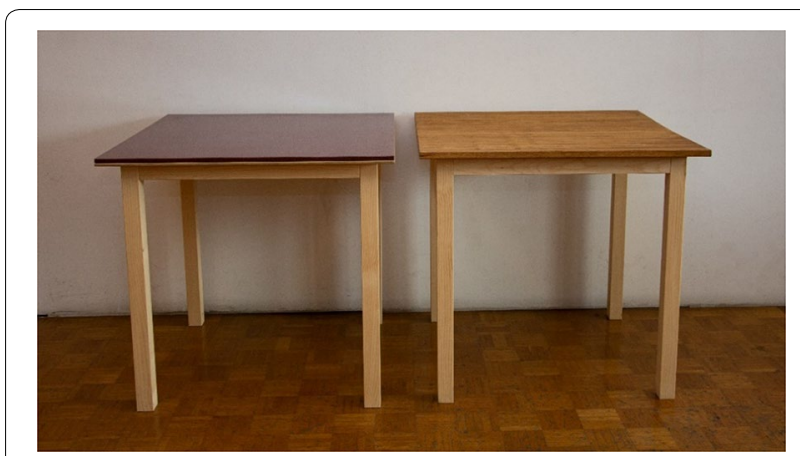

Fig. 2 Desks with top surfaces made of MFTC (left) and oak (right) has been shown to restore this core executive function [26]. In the task, a circle appeared on either the left or the right side of the screen. Participants were instructed to press the left arrow key when the circle was green and the right arrow key when the circle was red, regardless of the circle location on the screen. In approximately half of the trials, the location of the circle and the correct response key were congruent (e.g. a red circle appeared on the right) and in the other half they were incongruent. Each session consisted of 100 trials, taking about $90-120 \mathrm{~s}$ to complete.

\section{Subjective assessment of materials}

At each desk, subjects completed a short writing task and answered four questions examining their perception of a material. Results of the subjective assessment are not reported in this article.

\section{Arm and material temperature}

Arm and material temperatures were measured as part of the protocol using a non-invasive thermal camera. The results of the temperature measurement are not reported here.

\section{Experimental procedure}

The experimental (within-subject) procedure is outlined in Fig. 3. Before the first session, the necessary instructions and explanations were given to the subjects. It was explained to them how to rate the affective states (arousal and pleasure) and they completed a short demo of the cognitive task (Simon task). The instructions were also available in the computerized testing during all sessions. In addition, subjects were instructed not to communicate with each other at any stage of the experimental session and to abstain from caffeinated beverages prior to the testing.

The participants started with the baseline period, where they were brought to a control desk. They rested for $1 \mathrm{~min}$ in silence before completing the cognitive task and reporting affective states (CTAS; measurement time "Pre"), to minimize any influences of the previous interaction with the researcher(s). Subjects were instructed to keep their gaze at the desk surface during all rest periods throughout the experiment.

After the baseline period, participants began with the experimental part of the study, where they sat at a desk made of one out of 10 desk surface materials (the order was randomized). Before completing CTAS for the second time (measurement time "Start"), they again rested for $1 \mathrm{~min}$, (1) to enable dissipation of any temporary influences resulting from the relocation from the baseline period and (2) to enable subjects to gaze at the desk surface. 


\begin{tabular}{|c|c|c|c|c|c|c|c|}
\hline \multicolumn{2}{|c|}{ Baseline } & \multicolumn{6}{|c|}{ Experimental period } \\
\hline & Pre & & Start & & Post & & \\
\hline Rest & $\begin{array}{l}\text { Affect \& } \\
\text { Cognition }\end{array}$ & Rest & $\begin{array}{l}\text { Affect \& } \\
\text { Cognition }\end{array}$ & Rest & $\begin{array}{c}\text { Affect \& } \\
\text { Cognition }\end{array}$ & $\begin{array}{c}\text { Writing } \\
\text { task }\end{array}$ & $\begin{array}{c}\text { Material } \\
\text { rating }\end{array}$ \\
\hline $1 \mathrm{~min}$ & $3 \mathrm{~min}$ & $1 \mathrm{~min}$ & $3 \mathrm{~min}$ & $15 \mathrm{~min}$ & $3 \mathrm{~min}$ & $1 \mathrm{~min}$ & $1 \mathrm{~min}$ \\
\hline
\end{tabular}

!: Temperature measurement

Fig. 3 Experimental procedure

After this, participants rested for 15 min while keeping their bare arms immobile and flat on the desk and their gaze directed to the desk surface. After the rest period, subjects completed CTAS for the third and last time (measurement time "Post"). Participants then completed the short hand writing task and answered the four questions on subjective assessment of the material.

Subjects repeated the entire session 10 times, once for each desk material. They took 15-min breaks between sessions, when more than one was conducted in the same day. Up to five sessions per day were planned.

\section{Statistical analysis}

The data were processed and analysed in $\mathrm{R}$ 3.6.1 [37] using R Studio 1.2.1335 [38] with the packages dplyr [39], ggplot2 [40], rstatix [41], rcompanion [42], wmwpow [43], and WebPower [44]. Data from the entire sample of 16 participants were available and analysed in all results presented below. There were no missing values, as the computerized testing tool did not allow progressing through tasks without providing a response.

The assumptions of parametric tests were not met for any of the analysed outcomes, so we calculated bootstrapped medians and percentile confidence intervals to report observed values and their spread. We used nonparametric tests throughout the analysis.

To compare subjective assessment ratings between materials and examine differences between materials regarding changes in affective states and cognitive performance, we conducted Friedman tests that test for the difference between paired observations on ranked data. Separate Friedman tests were conducted for each subjective assessment item as a dependent variable and material as an independent variable. Similarly, separate Friedman tests comparing change scores (the difference in scores between two measurement times) between materials were conducted individually for each affective state and cognitive performance outcome, with the change score as the dependent variable and material as the independent variable. $p$ values in Friedman tests were adjusted with the Bonferroni correction.

To investigate changes in affective states and cognitive performance between study parts across all materials, we performed one-sample Wilcoxon signed rank tests that test for the difference between the observed values and a theoretical value, by comparing the ranks of the computed differences. Unless noted otherwise, $p$ values in multiple comparisons were adjusted with the Bonferroni correction. The significance threshold was set at 0.05 ; reported effect sizes are on the measurement scale.

\section{Results}

In most cases more than one subject participated in the study simultaneously (up to four). It generally took four visits (i.e. over a period of 4 days) for each participant to complete the study for all 10 desk materials; typically, less than a week passed between visits.

\section{Affective states}

Results on pleasure and arousal dimension across all materials are presented in Fig. 4 and Additional file 1: Table S1. The subjects generally reported feelings that were around the middle (or slightly higher) of the arousal and pleasure continuum.

Change scores (the difference in scores between two study parts) across materials in both affect dimensions were computed with Wilcoxon signed rank test and are presented in Table 2. Neither affective dimension significantly changed from the Pre to the Start period. In contrast, both arousal and pleasure scores decreased in the Post period when compared to either of the first two phases; the scores were approximately one point lower (on the 9-point scale) in the Post measurement time compared to the values taken in the Start and Pre phase. 

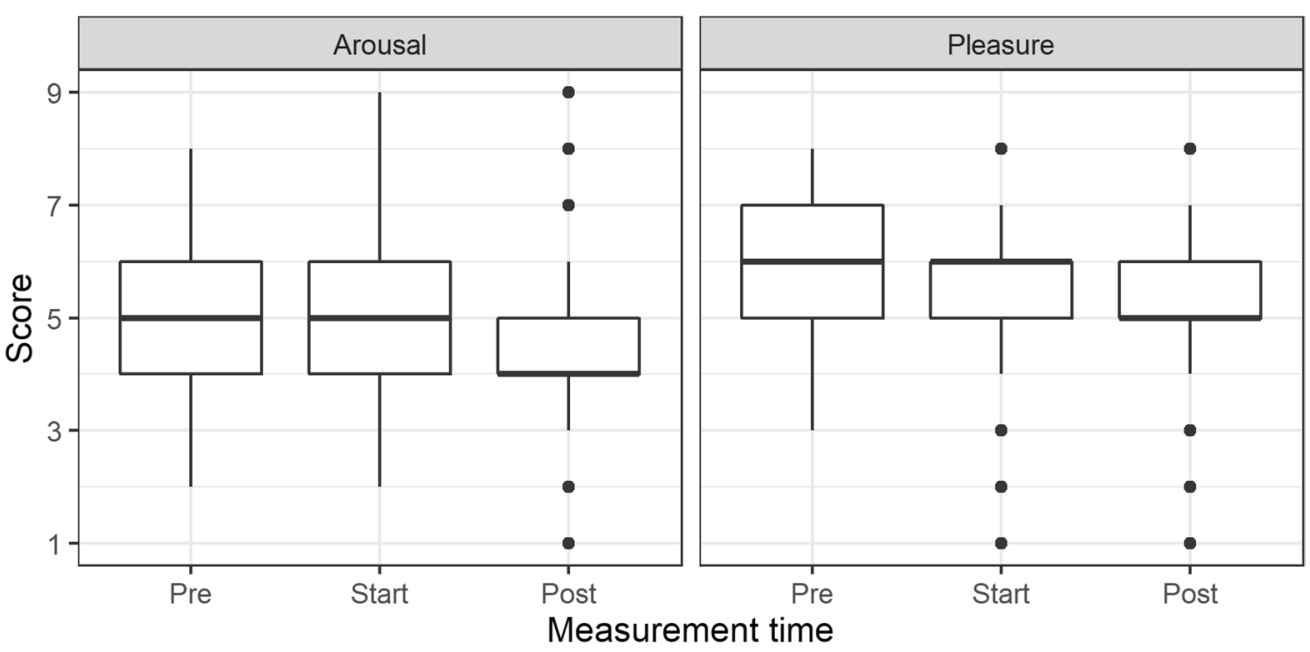

Fig. 4 Arousal and pleasure scores across all materials

Table 2 Changes in arousal and pleasure scores across study parts (all materials combined)—results of the Wilcoxon signed rank test

\begin{tabular}{llllll}
\hline Affect dimension & Change period & $\boldsymbol{V}$ & Pseudomedian & $\mathbf{9 5 \%} \mathrm{Cl}$ & $\boldsymbol{p}$ \\
\hline Pleasure & Start-Pre & 1057 & 0.000 & -0.000 to 0.000 & 1 \\
& Post-Pre & 1121 & -1.000 & -1.000 to -0.000 & $<0.001^{* * *}$ \\
& Post-Start & 737.5 & -1.000 & -1.000 to -0.500 & $<0.001^{* * *}$ \\
Arousal & Start-Pre & 982.0 & -0.000 & -1.000 to 0.000 & 0.438 \\
& Post-Pre & 1238 & -1.000 & -1.500 to -1.000 & $<0.001^{* * *}$ \\
& Post-Start & 608.0 & -1.000 & -1.500 to -1.000 & $<0.001^{* * *}$ \\
\hline
\end{tabular}

$p$-values are adjusted with the Bonferroni correction

$V, V$-statistic; $\mathrm{Cl}$, confidence interval. ${ }^{* * *} p<0.001$

The scores on both affective dimensions grouped by materials are displayed in Fig. 5 and Additional file 1: Tables S2 and S3. The results of Friedman tests comparing change scores in arousal and pleasure ratings between materials are presented in Table 3. There were no statistically significant results; neither arousal nor pleasure change scores differed between materials.

\section{Cognitive performance}

Three outcomes of the Simon task were analysed: (1) proportion of correct answers, (2) mean response time on correct answers, and (3) Simon effect, the difference in mean response time between correct answers on incongruent trials and correct answers on congruent trials (higher values generally suggest lower cognitive inhibition). The results across all materials are presented in Fig. 6 and Additional file 1: Table S4. Typically, subjects responded correctly to more than $90 \%$ of the trials, their mean response time in correct answers was around $400 \mathrm{~ms}$, and the Simon effect was usually between 20 and $30 \mathrm{~ms}$.
Change scores in the three Simon task outcomes computed with Wilcoxon signed rank test (across all materials) are presented in Table 4. The proportion of correct answers in the Simon task was higher in the Start period when compared to the Pre period by approximately one percentage point, while no differences were detected between other study parts. Differences in the other two Simon task outcomes between study parts were not detected.

The scores on the Simon task outcomes grouped by materials are presented in Fig. 7 and Additional file 1: Tables S5-S7. The results of Friedman tests comparing change scores in Simon task outcomes are presented in Table 5. No statistically significant differences were detected; change scores did not differ between materials.

\section{Discussion}

The results did not support our first group of hypotheses; affective states did not differ between wooden and non-wooden desks. When pooled data were inspected (combining all materials), neither arousal nor pleasure 


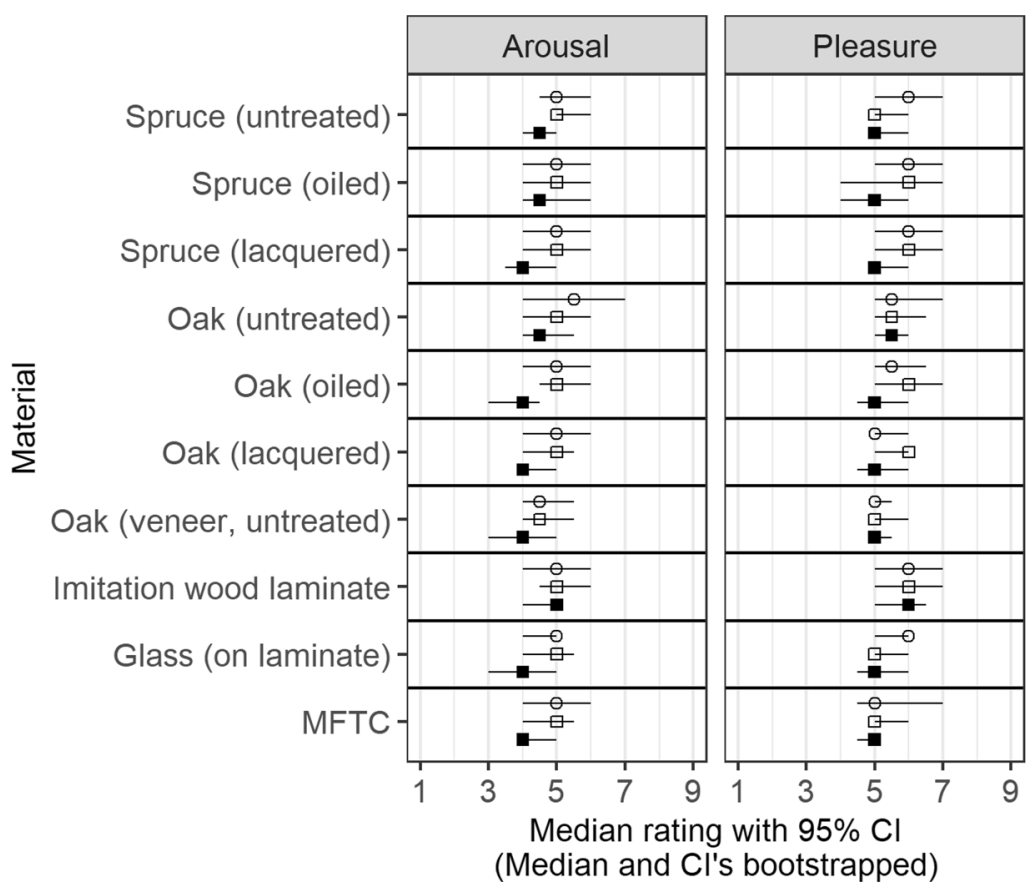

Part of the study

- Pre

口 Start

- Post

Fig. 5 Arousal and pleasure scores grouped by materials and measurement time

Table 3 Changes in arousal and pleasure across measurement times with material as a dependent variable-results of the Friedman tests

\begin{tabular}{llllll}
\hline Affect dimension & Change period & df & $\boldsymbol{x}^{\mathbf{2}}$ & Kendall's $\boldsymbol{W}$ & $\boldsymbol{p}$ \\
\hline Pleasure & Start-Pre & 9 & 15.54 & 0.108 & 0.232 \\
& Post-Pre & 9 & 5.033 & 0.035 & 1 \\
\multirow{3}{*}{ Arousal } & Post-Start & 9 & 6.899 & 0.048 & 1 \\
& Start-Pre & 9 & 2.322 & 0.016 & 1 \\
& Post-Pre & 9 & 5.762 & 0.040 & 1 \\
& Post-Start & 9 & 5.163 & 0.036 & 1
\end{tabular}

$p$-values are adjusted with the Bonferroni correction

$\mathrm{df}$, degrees of freedom, $x^{2}$, Chi-square value

significantly changed from the Pre to the Start period, but ratings on both dimensions were lower in the Post sessions. These scores suggest that participants were experiencing more feelings such as sleepiness, tiredness, or boredom in the last stage of the experiment. It should be pointed out, however, that the differences in affective state scores were small; overall, the arousal and pleasure states of participants were relatively similar in Pre, Start, and Post measurement times. This lack of substantial differences is not surprising, given that participants rested for only a short period of time while being relatively unstimulated. The reason that we were able to detect these small changes might have been related to using two single-item (arousal and pleasure) scales instead of often used longer questionnaires that can be less sensitive in restoration studies (e.g. POMS). For example, a recent study from Demattè and colleagues [18] administered Positive and Negative Affect Schedule (PANAS) before and after the exposure to a wooden setting and did not detect any changes between the measurements, despite the study's large sample (102 participants), relatively large amount of wood coverage, and the presence of olfactory stimulation. While it is possible that the subjects' affective states did not significantly change between the measurements, it could be that PANAS, consisting of many items capturing specific affective phenomena, might have missed broad and subtle changes that could have been detected with measures of core affect, including the one used in this study. Such measures may be more sensitive in detecting changes with small effect sizes that can have large effects in practice, due to small, seemingly insignificant effects compounding over time.

The observed trend of decreasing arousal and pleasure did not differ between materials-wooden materials did not seem to influence the affective states, as we expected. Similar results were observed in the two studies by Tsunetsugu et al. [8, 22], where differences in affective states were not detected between the test settings differing in the amount of incorporated wood. However, certain studies did detect an effect of wood exposure on affective states. Compared to the present 

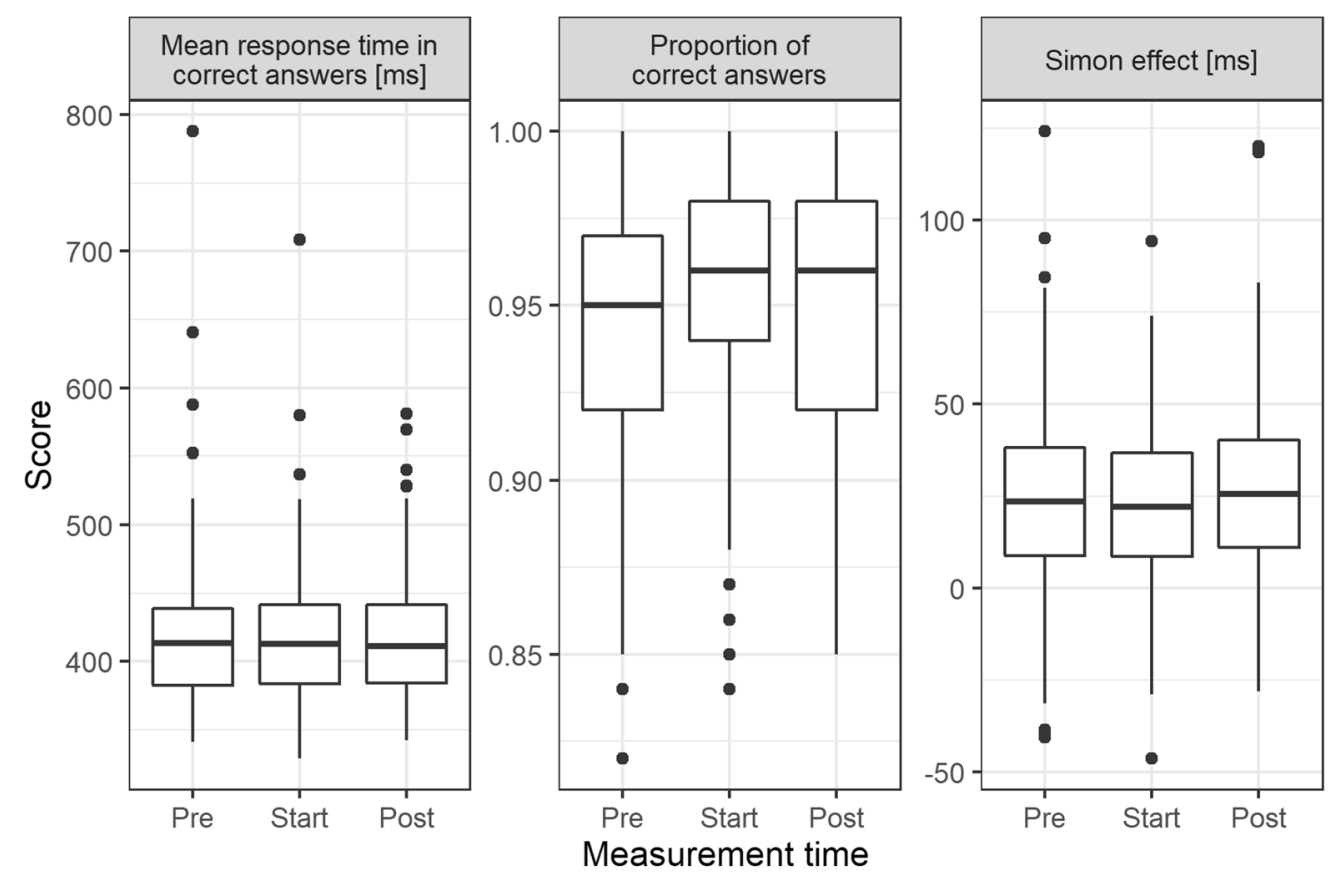

Fig. 6 Simon task outcomes across all materials

Table 4 Changes in Simon task outcomes across study parts (all materials combined)—results of Wilcoxon signed rank test

\begin{tabular}{|c|c|c|c|c|c|}
\hline Simon task & Change period & $v$ & Pseudomedian & $95 \% \mathrm{Cl}$ & $p$ \\
\hline \multirow[t]{3}{*}{ Proportion of correct answers } & Start-Pre & 5931 & 0.010 & 0.005 to 0.015 & $<0.001^{* * *}$ \\
\hline & Post-Pre & 5034 & 0.005 & -0.000 to 0.010 & 0.141 \\
\hline & Post-Start & 3872 & -0.005 & -0.010 to -0.000 & 0.107 \\
\hline \multirow{3}{*}{$\begin{array}{l}\text { Mean response time on correct } \\
\text { answers (ms) }\end{array}$} & Start-Pre & 6187 & -0.785 & -4.435 to 2.785 & 1 \\
\hline & Post-Pre & 6619 & 0.667 & -3.140 to 4.470 & 1 \\
\hline & Post-Start & 7126 & 1.985 & -1.355 to 5.305 & 0.730 \\
\hline \multirow[t]{3}{*}{ Simon effect (ms) } & Start-Pre & 5852 & -2.085 & -6.190 to 2.055 & 0.951 \\
\hline & Post-Pre & 7143 & 2.965 & -1.975 to 7.980 & 0.693 \\
\hline & Post-Start & 7123 & 2.810 & -1.805 to 7.735 & 0.736 \\
\hline
\end{tabular}

$p$-values are adjusted with the Bonferroni correction

$V, V$-statistic; $\mathrm{Cl}$, confidence interval, ${ }^{* * *} p<0.001$

study, these incorporated a larger amount of wood in their test environments [20] and those who used solid wood also had detectable levels of wood scents in the air $[18,19]$. Other studies that observed lowered physiological arousal in the wooden settings similarly incorporated a relatively large amount of wood in their test settings (without olfactory stimulation). Although these studies did not directly measure affective states, lower physiological arousal (when considered a marker of lower stress levels) would likely be reflected in changes in affective phenomena [21, 45, 46]. Interestingly, in both studies decreased physiological arousal was observed early in the study and it did not seem to depend on the exposure time, nor was it evident only after the stress-inducing activity.

Put together, these results suggest that the amount of wood coverage might be one of the prime suspects for the diverging results of the present study and other findings. Indeed, according to stress reduction theory and attention restoration theory, environments that are generally richer in natural stimuli are more to likely to benefit humans $[2,4]$. Perhaps the small desk surface was not stimulating enough to generate these benefits, despite participants being instructed to keep their gaze 


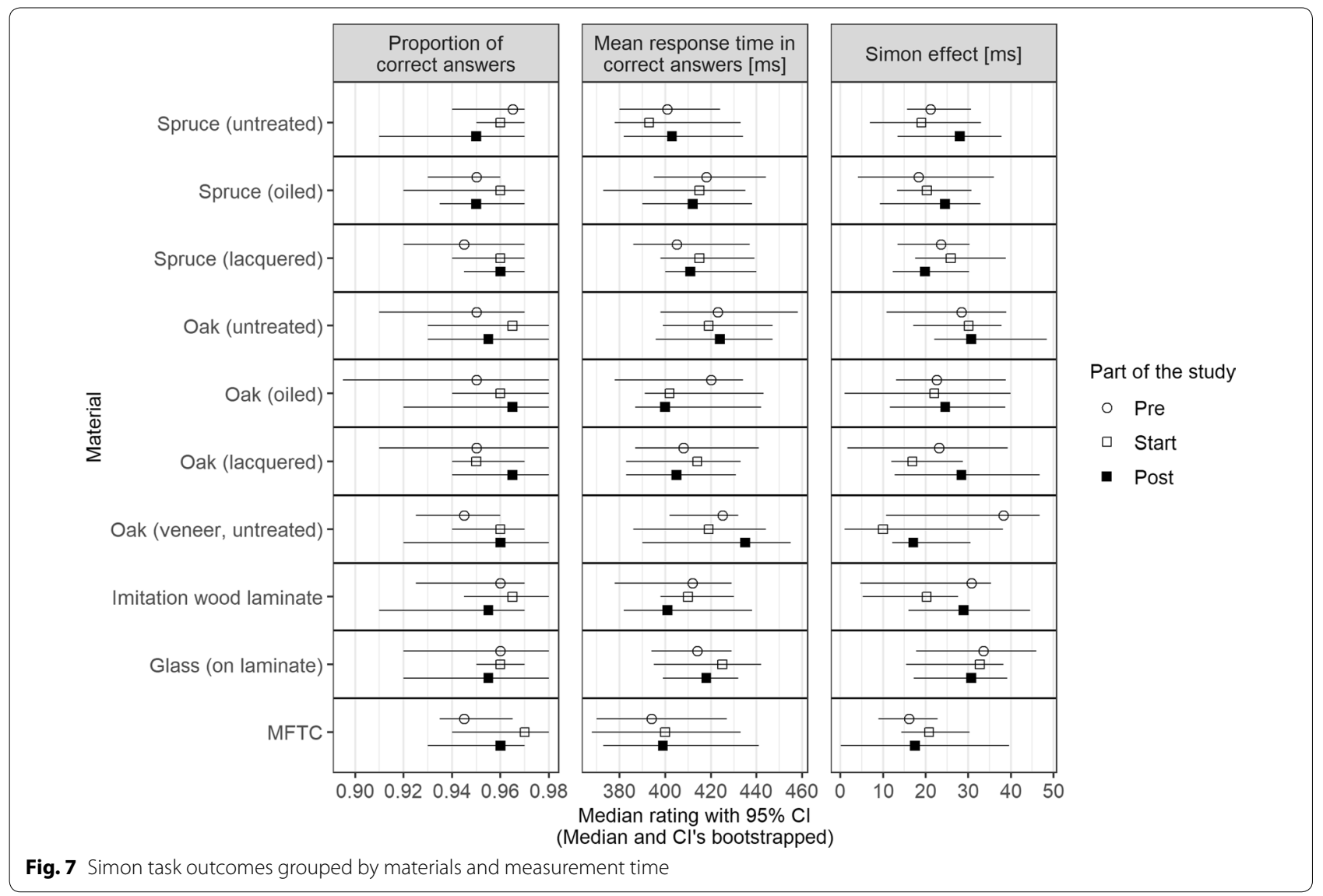

Table 5 Changes in Simon task outcomes across measurement times with material as a dependent variable-results of the Friedman tests

\begin{tabular}{llllll}
\hline Simon task & Change period & df & $\boldsymbol{x}^{\mathbf{2}}$ & Kendall's $\boldsymbol{W}$ & $\boldsymbol{p}$ \\
\hline Proportion & Start-Pre & 9 & 2.929 & 0.020 & 1 \\
$\begin{array}{l}\text { of correct } \\
\text { answers }\end{array}$ & Post-Pre & 9 & 9.308 & 0.065 & 1 \\
Mean & Post-Start & 9 & 8.912 & 0.062 & 1 \\
response & Start-Pre & 9 & 13.39 & 0.093 & 0.437 \\
time on cor- & Post-Pre & 9 & 6.709 & 0.047 & 1 \\
rect answers & Post-Start & 9 & 7.173 & 0.050 & 1 \\
Simon effect & Start-Pre & 9 & 6.600 & 0.046 & 1 \\
& Post-Pre & 9 & 9.055 & 0.063 & 1 \\
& Post-Start & 9 & 4.486 & 0.031 & 1 \\
\hline
\end{tabular}

$p$-values are adjusted with the Bonferroni correction

$\mathrm{df}$, degrees of freedom, $x^{2}, \mathrm{Chi}$-square value

at the material throughout the experiment. It is also possible that such less intense environmental stimulations might benefit people, but that these benefits would become apparent only during the recovery following an induction of stress or fatigue [26]. Exposure to a larger amount of wood coverage combined with a stress- or fatigue-inducing activity would likely increase the chances of detecting potential effects of wood exposure on human affective states.

The results were similarly not in line with our second group of hypotheses; we did not observe differences in cognitive performance between materials. Across all materials, there were no significant differences between the study periods in mean response time in correct answers or in the Simon effect; in contrast, the proportion of correct answers was higher in the Start period than in the Pre phase. Considering that the two cognitive task sessions were conducted with only a few min in between, perhaps the first session served as a training experience that improved the results on the second administration. Alternatively, the results could have improved due to other differences between the first and the second session; for example, due to the participants' change from the standing to the sitting position or due to fewer distractions that the subjects faced in the second session, as they were isolated from each other.

As was the case in the examination of affective states, we did not find any differences in cognitive performance between the tested desk materials. Only two existing studies tested the effects of wood exposure on 
cognitive performance; one did not report the results [17] and the other did not observe any differences between the wooden and non-wooden environment [16]. Despite our results being in line with the latter finding, they run counter to the findings observed in several other studies with somewhat similar research protocols, which mainly differ by incorporating other elements of nature instead of wood [1]. While wood may not exhibit attention enhancing properties similar to other elements of nature, it is also possible that other factors played a role.

In all wood exposure studies (including the present study) cognitive fatigue might not have been sufficiently induced. Attention restoration theory is specific to predict restoration from induced attention fatigue but not improvement in cognitive capabilities, if these are not fatigued prior to the exposure to natural environments. However, several studies found that exposure to nature improved cognitive performance even without prior induction of attention fatigue, suggesting that other mechanisms, such as changes in affective states, may be important [26]. Another reason for the absence of effects on cognitive performance may be related to the cognitive task we deployed. It has been proposed that cognitive tasks with certain properties are more likely to capture the differences in performance in attention restoration studies. Among other qualities, tasks should be high in cognitive demand, which may not have been the case in this study. Namely, the percentage of correct answers in the Simon task was often in the high nineties, with several sessions where all the answers were correct. Furthermore, the results generally improved on the second administration of the task, suggesting that the employed task was not sufficiently difficult to lead to attention fatigue after the first administration. Our findings urge future studies to employ tasks demanding enough to induce attention fatigue and to avoid the ceiling effect, where the range of the scores is restricted and prevents potential differences between the environments to occur.

\section{Limitations}

The sample size in the study was relatively small and the statistical power was too low for the study to reliably capture small and moderate effects that could be practically relevant. In addition, the study did not include any physiological measures that could capture important changes that would not necessarily be detected in either the measures of affective states or the tests of cognitive performance. Several confounders could have influenced the results that might not have been sufficiently controlled for, including the frequency of testing sessions, time and day of testing, and the potential effects of being without caffeine before and during the test.

\section{Conclusions}

The findings suggest that a brief visual and tactile exposure to a wooden small desk surface might not be enough to significantly improve human affective states and cognitive performance, even though the study tested a variety of materials and included a sensitive measure of affective states. To increase the chances of capturing potential effects, future studies should include a larger number of participants, increase the amount of wood coverage, and incorporate a cognitive task with different properties, including higher cognitive demands. Although the study did not observe positive outcomes following wood exposure, it is important for the research in this field to continue, as even small effects of wood stimulation could contribute to substantial real-life outcomes. Wood furnishings are relatively simple and inexpensive to implement on a wide-scale, while at the same time wood exposure does not depend on human effort and determination, as do many other psychosocial interventions addressing stress and well-being.

\section{Supplementary information}

Supplementary information accompanies this paper at https://doi. org/10.1186/s10086-020-01890-3.

Additional file 1: Table S1. Arousal and pleasure scores across all desk materials. Table S2. Arousal scores across study parts for each desk material. Table S3. Pleasure scores across study parts for each desk material. Table S4. Simon task outcomes across all desk materials. Table S5. Proportion of correct answers in the Simon task across study parts for each desk material. Table S6. Mean response time [ms] on correct answers in the Simon task across study parts for each desk material. Table S7. Simon effect [ms] in the Simon task across study parts for each desk material. Figure S1. Engineered stone. Figure S2. Imitation wood laminate. Figure S3. Oak (lacquered). Figure S4. Oak (oiled). Figure S5. Oak (untreated). Figure S6. Oak (veneer, untreated). Figure S7. Spruce (lacquered). Figure S8. Spruce (oiled). Figure S9. Spruce (untreated).

\section{Abbreviations}

CTAS: Cognitive task and (reporting) affective states; MFTC: Mineral-filled thermoplastic composite; POMS: Profile of Mood States; SD: Standard deviation.

\section{Acknowledgements}

The authors gratefully acknowledge the European Commission for funding the InnoRenew CoE project (Grant Agreement 739574) under the Horizon2020 Widespread-Teaming program, the Republic of Slovenia (Investment funding of the Republic of Slovenia and the European Union of the European Regional Development Fund), and the Slovenian Research Agency ARRS for funding infrastructure program 10-0035.

\section{Authors' contributions}

All authors contributed to preparing the research protocol. DL and NP collected the data; DL and MB analysed the data. DL prepared a large part of the article content with major contributions from $\mathrm{MB}$ and significant contributions from NŠ. All authors read and approved the final manuscript. 


\section{Funding}

The study is part of the InnoRenew project (Horizon2020 Widespread-Teaming program Grant Agreement 739574), was supported by the Republic of Slovenia (Investment funding of the Republic of Slovenia and the European Union of the European Regional Development Fund), and the Slovenian Research Agency (ARRS) for funding infrastructure program 10-0035

\section{Availability of data and materials}

All data generated or analysed during this study are included in this published article and its supplementary information files.

\section{Ethics approval and consent to participate}

The study protocol and testing procedures (ClinicalTrials.gov identifier: NCT03733366) were approved by the National Medical Ethics Committee of Slovenia (No. 0120-631/2017/2) and the research was carried out in compliance with the Oviedo convention. Participants signed an approved informed consent waiver that provided them information about study purpose and procedure, participant rights, and data management.

\section{Consent for publication}

Participants agreed the collected data can be used for research purposes, including publishing.

\section{Competing interests}

The authors declare that they have no competing interests.

\section{Author details}

${ }_{1}^{1}$ InnoRenew CoE, Livade 6, 6310 Izola, Slovenia. ${ }^{2}$ Andrej Marušič Institute, University of Primorska, Muzejski trg 2,6000 Koper, Slovenia. ${ }^{3}$ Faculty of Health Sciences, University of Primorska, 6310 Izola, Slovenia.

Received: 21 April 2020 Accepted: 28 May 2020

Published online: 08 June 2020

\section{References}

1. Mcsweeney J, Rainham D, Johnson SA, Sherry SB, Singleton J (2015) Indoor nature exposure (INE): a health-promotion framework. Health Promot Int 30:126-139. https://doi.org/10.1093/heapro/dau081

2. Ulrich RS (1983) Aesthetic and affective response to natural environment. Behav Nat Environ Adv Theory Res 6:85-125. https://doi. org/10.1007/978-1-4613-3539-9

3. Kaplan R, Kaplan S (1989) The experience of nature: a psychological perspective, vol 6. Cambridge Univ Press, Cambridge. https://doi. org/10.1037/030621

4. Kaplan S (1995) The restorative benefits of nature: toward an integrative framework. J Environ Psychol 15:169-182. https://doi.org/10.1016/02724944(95)90001-2

5. Klepeis NE, Nelson WC, Ott WR, Robinson JP, Tsang AM, Switzer P, Behar JV, Hern FSC, Engelmann WH (2001) The National Human Activity Pattern Survey (NHAPS): a resource for assessing exposure to environmental pollutants. J Expo Sci Environ Epidemiol 11:231-252. https://doi. org/10.1038/sj.jea.7500165

6. Rice J, Kozak RA, Meitner MJ, Cohen DH (2006) Appearance wood products and psychological well-being. Wood Fiber Sci 38:644-659

7. Burnard MD, Nyrud AQ, Bysheim K, Kutnar A, Vahtikari K, Hughes M (2017) Building material naturalness: perceptions from Finland, Norway and Slovenia. Indoor Built Environ 26:92-107. https://doi.org/10.1177/14203 26X15605162

8. Tsunetsugu Y, Miyazaki Y, Sato H (2007) Physiological effects in humans induced by the visual stimulation of room interiors with different wood quantities. J Wood Sci 53:11-16. https://doi.org/10.1007/s1008 6-006-0812-5

9. Ikei H, Song C, Miyazaki Y (2017) Physiological effects of wood on humans: a review. J Wood Sci 63:1-23. https://doi.org/10.1007/s1008 6-016-1597-9

10. Burnard MD, Kutnar A (2015) Wood and human stress in the built indoor environment: a review. Wood Sci Technol 49:969-986. https://doi. org/10.1007/s00226-015-0747-3
11. Nyrud AQ, Bringslimark T (2010) Is interior wood use psychologically beneficial? A review of psychological responses toward wood. Wood Fiber Sci 42:202-218

12. Brown SK (1999) Chamber assessment of formaldehyde and VOC emissions from wood-based panels. Indoor Air 9:209-215. https://doi. org/10.1111/j.1600-0668.1999.t01-1-00008.x

13. Ikei H, Song C, Miyazaki Y (2018) Physiological effects of touching hinoki cypress (Chamaecyparis obtusa). J Wood Sci 64:226-236. https://doi. org/10.1007/s10086-017-1691-7

14. Sakuragawa S, Kaneko T, Miyazaki Y (2008) Effects of contact with wood on blood pressure and subjective evaluation. J Wood Sci 54:107-113. https://doi.org/10.1007/s10086-007-0915-7

15. Zhang $X$, Lian Z, Wu Y (2017) Human physiological responses to wooden indoor environment. Physiol Behav 174:27-34. https://doi.org/10.1016/j. physbeh.2017.02.043

16. Fell DR (2010) Restorative properties of wood in the built indoor environment. Doctoral dissertation, University of British Columbia, Vancouver https://doi.org/10.14288/1.0071305

17. Burnard MD, Kutnar A (2019) Human stress responses in officelike environments with wood furniture. Build Res Inf. https://doi. org/10.1080/09613218.2019.1660609

18. Demattè ML, Zucco GM, Roncato S, Gatto P, Paulon E, Cavalli R, Zanetti $M$ (2018) New insights into the psychological dimension of woodhuman interaction. Eur J Wood Wood Prod 76:1093-1100. https://doi. org/10.1007/s00107-018-1315-y

19. Zhang X, Lian Z, Ding Q (2016) Investigation variance in human psychological responses to wooden indoor environments. Build Environ 109:58-67. https://doi.org/10.1016/j.buildenv.2016.09.014

20. Sakuragawa S, Miyazaki Y, Kaneko T, Makita T (2005) Influence of wood wall panels on physiological and psychological responses. J Wood Sci 51:136-140. https://doi.org/10.1007/s10086-004-0643-1

21. Parsons R, Tassinary LG (2002) Environmental psychophysiology. Handbook of environmental psychology. Wiley, Hoboken, pp 172-190

22. Tsunetsugu Y, Yoshifumi M, Sato H (2005) Visual effects of interior design in actual-size living rooms on physiological responses. Build Environ 40:1341-1346. https://doi.org/10.1016/j.buildenv.2004.11.026

23. Ikei H, Song C, Miyazaki Y (2017) Physiological effects of touching coated wood. Int J Environ Res Public Health 14:773. https://doi.org/10.3390/ ijerph 14070773

24. Ekkekakis P, Zenko Z (2016) Measurement of affective responses to exercise: from "affectless arousal" to "the most well-characterized" relationship between the body and affect. In: Meiselman HL (ed) Emotion measurement. Elsevier, Amsterdam, pp 299-321

25. Stone AA (1995) Measurement of affective response. In: Cohen S, Kessler RC, Gordon LU (eds) Measuring stress: a guide for health and social scientists. Oxford University Press, New York, pp 148-171

26. Stevenson MP, Schilhab T, Bentsen P (2018) Attention restoration theory II: a systematic review to clarify attention processes affected by exposure to natural environments. J Toxicol Environ Health Part B Crit Rev 21:227-268. https://doi.org/10.1080/10937404.2018.1505571

27. Hartig T, Staats $\mathrm{H}$ (2006) The need for psychological restoration as a determinant of environmental preferences. J Environ Psychol 26:215-226

28. Ohly H, White MP, Wheeler BW, Bethel A, Ukoumunne OC, Nikolaou V, Garside R (2016) Attention restoration theory: a systematic review of the attention restoration potential of exposure to natural environments. J Toxicol Environ Health Part B Crit Rev 19:305-343. https://doi. org/10.1080/10937404.2016.1196155

29. Prajapati B, Dunne M, Armstrong R (2010) Sample size estimation and power analysis. Optom Today. https://www.researchgate.net/profile/ Zahra_Almaspoor/post/How_to_use_G-power_software_for_calculatin g_my_sample_size/attachment/5a427a8c4cde-266d587dd456/AS:57585 6045195264@1514306188473/download/Gpower_tutorial_Praja pati_2010-.pdf. Accessed 16 Sept 2019

30. Mollan KR, Trumble IM, Reifeis SA, Ferrer O, Bay CP, Baldoni PL, Hudgens MG (2019) Exact power of the rank-sum test for a continuous variable. https://arxiv.org/abs/1901.04597. Accessed 16 Sept 2019

31. von Bastian CC, Locher A, Ruflin M (2013) Tatool: a Java-based opensource programming framework for psychological studies. Behav Res Methods 45:108-115. https://doi.org/10.3758/s13428-012-0224-y 
32. Russell JA, Weiss A, Mendelsohn GA (1989) Affect grid: a single-item scale of pleasure and arousal. J Pers Soc Psychol 57:493-502. https://doi. org/10.1037/0022-3514.57.3.493

33. Ekkekakis P (2008) Affect circumplex redux: the discussion on its utility as a measurement framework in exercise psychology continues. Int Rev Sport Exerc Psychol 1:139-159. https://doi.org/10.1080/1750984080 2287200

34. Killgore WDS (1998) The affect grid: a moderately valid, nonspecific measure of pleasure and arousal. Psychol Rep 83:639-642. https://doi. org/10.2466/pro.1998.83.2.639

35. Diamond A (2013) Executive functions. Annu Rev Psychol 64:135-168. https://doi.org/10.1146/annurev-psych-113011-143750

36. Hommel B (2011) The Simon effect as tool and heuristic. Acta Psychol 136:189-202. https://doi.org/10.1016/J.ACTPSY.2010.04.011

37. R Core Team (2019) R: a language and environment for statistical computing. R Foundation for Statistical Computing, Vienna. https://www.R-proje ct.org/. Accessed 16 Sept 2019

38. RStudio Team (2019) RStudio: integrated development environment for R. http://www.rstudio.com/. Accessed 16 Sept 2019

39. Wickham H, François R, Henry L, Müller K (2019) dplyr: a grammar of data manipulation. R package version 0.8.3. https://cran.r-project.org/packa ge $=$ dplyr. Accessed 16 Sept 2019

40. Wickham H, Chang W, Henry L, Pedersen TL, Takahashi K, Wilke C, Woo K, Yutani H (2019) ggplot2: create elegant data visualisations using the grammar of graphics. R package version 3.2.1. https://cran.r-project.org/ package $=$ ggplot2. Accessed 16 Sept 2019
41. Kassambara A (2019) rstatix: pipe-friendly framework for basic statistical tests. R package version 0.2.0. https://cran.r-project.org/package=rstatix. Accessed 16 Sept 2019

42. Mangiafico S (2019) rcompanion: functions to support extension education program evaluation. R package version 2.3.7. https://cran.r-proje ct.org/package=rcompanion

43. Trumble I, Ferrer O, Bay C, Mollan K (2019) wmwpow: precise and accurate power of the Wilcoxon-Mann-Whitney rank-sum. R package version 0.1.2. https://cran.r-project.org/package=wmwpow. Accessed $16 \mathrm{Sept}$ 2019

44. Zhang Z, Mai Y (2018) WebPower: basic and advanced statistical power analysis. R package version 0.5.2. https://cran.r-project.org/packa ge=WebPower. Accessed 16 Sept 2019

45. Hellhammer DH, Wüst S, Kudielka BM (2009) Salivary cortisol as a biomarker in stress research. Psychoneuroendocrinology 34:163-171. https ://doi.org/10.1016/J.PSYNEUEN.2008.10.026

46. Cohen S, Kessler RC, Gordon LU (1995) Strategies for measuring stress in studies of psychiatric and physical disorders. Measuring stress: a guide for health and social scientists. Oxford University Press, New York, pp 3-26

\section{Publisher's Note}

Springer Nature remains neutral with regard to jurisdictional claims in published maps and institutional affiliations.

\section{Submit your manuscript to a SpringerOpen ${ }^{\circ}$ journal and benefit from:}

- Convenient online submission

- Rigorous peer review

- Open access: articles freely available online

- High visibility within the field

- Retaining the copyright to your article

Submit your next manuscript at $\boldsymbol{\Delta}$ springeropen.com 